\title{
Growth characteristics in individuals with osteogenesis imperfecta in North America: results from a multicenter study
}

\author{
Mahim Jain, MD, $\mathrm{PhD}^{1,2}$, Allison Tam, MD ${ }^{1}$, Jay R. Shapiro, $\mathrm{MD}^{2}$, Robert D. Steiner, $\mathrm{MD}^{3,4}$, \\ Peter A. Smith, MD ${ }^{5}$, Michael B. Bober, $\mathrm{MD}^{6}$, Tracy Hart, $\mathrm{BS}^{7}$, David Cuthbertson, $\mathrm{MS}^{8}$, \\ Jeff Krischer, $\mathrm{PhD}^{8}$, Mary Mullins, BSN, RN ${ }^{1}$, Sunil Bellur, BA ${ }^{1}$, Peter H. Byers, $\mathrm{MD}^{9}$, \\ Melanie Pepin, MS, LGC ${ }^{9}$, Michaela Durigova, $\mathrm{PhD}^{10}$, Francis $\mathrm{H}$. Glorieux, MD, PhD ${ }^{10}$, \\ Frank Rauch, MD ${ }^{10}$, Brendan Lee, MD, PhD ${ }^{1,11}$, \\ V. Reid Sutton, $\mathrm{MD}^{1,11}$, Members of the Brittle Bone Disorders Consortium*, and \\ Sandesh C. S. Nagamani, MD ${ }^{1,11}$
}

\begin{abstract}
Purpose: Osteogenesis imperfecta (OI) predisposes people to recurrent fractures, bone deformities, and short stature. There is a lack of large-scale systematic studies that have investigated growth parameters in OI.

Methods: Using data from the Linked Clinical Research Centers, we compared height, growth velocity, weight, and body mass index (BMI) in 552 individuals with OI. Height, weight, and BMI were plotted on Centers for Disease Control and Prevention normative curves.

Results: In children, the median z-scores for height in OI types I, III, and IV were $-0.66,-6.91$, and -2.79 , respectively. Growth velocity was diminished in OI types III and IV. The median z-score for weight in children with OI type III was -4.55 . The median $z_{-}$ scores for BMI in children with OI types I, III, and IV were 0.10 , 0.91 , and 0.67 , respectively. Generalized linear model analyses
\end{abstract}

demonstrated that the height $\mathrm{z}$-score was positively correlated with the severity of the OI subtype $(P<0.001)$, age, bisphosphonate use, and rodding $(P<0.05)$.

Conclusion: From the largest cohort of individuals with OI, we provide median values for height, weight, and BMI z-scores that can aid the evaluation of overall growth in the clinic setting. This study is an important first step in the generation of OI-specific growth curves.

Genetics in Medicine (2019) 21:275-283; https://doi.org/10.1038/s41436018-0045-1

Key Words: Growth; Height; Natural history study; Osteogenesis imperfecta; Weight

\section{INTRODUCTION}

Osteogenesis imperfecta (OI) - a group of Mendelian disorders of connective tissue (MIM 166200, 166210, 259420, 166220, 610967, 613982, 610682, 610915, 259440, 613848, 610968, $609220,614856,613849,615066,615220,616229,616507)-$ predisposes people to recurrent fractures, bone deformities, and short stature. ${ }^{1}$ Nearly $90 \%$ of individuals with OI have pathogenic variants in COL1A1 and COL1A2 that encode for the $\alpha 1$ and $\alpha 2$ chains of type I collagen-a major protein of the bone matrix. ${ }^{2}$ Over the past decade, the discovery of numerous genes as causes for OI has underscored the genetic heterogeneity of this disorder. However, the Sillence classification, which was proposed well before the genetic basis of OI was known, continues to be used for management and counseling purposes. ${ }^{3}$ Accordingly, the autosomal dominant, type-Icollagen-related OI is classified into nondeforming (type I), perinatally lethal (type II), progressively deforming (type III), and common variable (type IV) forms.

\footnotetext{
${ }^{1}$ Department of Molecular and Human Genetics, Baylor College of Medicine, Houston, Texas, USA; ${ }^{2}$ Department of Bone and Osteogenesis Imperfecta, Kennedy Krieger Institute, Baltimore, Maryland, USA; ${ }^{3}$ Departments of Pediatrics and Molecular and Medical Genetics, Oregon Health and Science University, Portland, Oregon, USA; ${ }^{4}$ University of Wisconsin School of Medicine and Public Health, Madison, Wisconsin, USA; ${ }^{5}$ Shriners Hospitals for Children, Chicago, Illinois, USA; ${ }^{6}$ Division of Medical Genetics, Alfred I. duPont Hospital for Children, Wilmington, Delaware, USA; ${ }^{7}$ Osteogenesis Imperfecta Foundation, Gaithersburg, Maryland, USA; ${ }^{8}$ College of Medicine, University of South Florida, Tampa, Florida, USA; ${ }^{9}$ Departments of Medicine and Pathology, Division of Medical Genetics, University of Washington, Seattle, Washington, USA; ${ }^{10}$ Shriners Hospital for Children, Canada and McGill University, Montreal, Quebec, Canada; ${ }^{11}$ Texas Children’s Hospital, Houston, Texas, USA. Correspondence: Sandesh C.S. Nagamani Nagamani@bcm. edu)
}

Brittle Bone Disorders Consortium are listed after the Acknowledgments section.

Members of the Brittle Bone Disorders Consortium are listed in the acknowledgements.

These authors contributed equally: Mahim Jain, Allison Tam.

These authors contributed equally: V. Reid Sutton, Sandesh C. S. Nagamani. 
Short stature is a hallmark of the moderate-to-severe forms of OI. Previous studies have demonstrated that the mean birth length and weight in individuals with OI are below the normative data from the general population. ${ }^{4,5}$ Whereas final height is significantly restricted in those with OI types III and IV, even those with milder forms of disease (i.e., type I), have reductions in overall height compared with the general population. $^{5-8}$ To date, few studies have systematically analyzed the growth parameters in OI. Most reports are limited either by the size of the cohort or the lack of data on adults with $\mathrm{OI}^{5,6,8-13}$. In fact, there are only two studies outlining the growth parameters in both children and adults with OI. ${ }^{7,8}$ The largest cohort of both children and adults with OI described to date (144 children and 199 adults) revealed that, in addition to decreased height, obesity is common in OI. ${ }^{7}$ Detailed assessments of anthropometric measures in OI can have both diagnostic and management implications. Appropriate characterization of the growth parameters can help identify affected individuals who are not meeting the expected growth patterns and institute lifestyle modifications for weight control.

Here, we analyzed the cross-sectional growth parameters in a large cohort of individuals with OI from across North America ( $n=552 ; 408$ children and 144 adults) who were enrolled in the observational study "Longitudinal Study of Osteogenesis Imperfecta" that was conducted by the OI Linked Clinical Research Centers (LCRC). ${ }^{14}$ The large sample size of the cohort allowed us to examine growth parameters in the various subtypes of OI, and assess the potential correlations between height and the type of OI, surgical rodding, and bisphosphonate use.

\section{MATERIALS AND METHODS}

\section{Study population}

Details of the LCRC and Longitudinal Study of Osteogenesis Imperfecta have been described previously. ${ }^{14}$ The LCRC comprised five clinical research centers: Baylor College of Medicine (Houston, TX); Kennedy Krieger Institute (Baltimore, MD) in collaboration with Nemours/Alfred I. DuPont Hospital for Children (Wilmington, DE); Oregon Health and Science University (Portland, OR); Shriners Hospital for Children (Chicago, IL); and Shriners Hospital for Children (Montreal, QC). The data collected from these clinical sites were coordinated and managed by the National Institutes of Health Rare Disease Clinical Research Network's Data Management and Coordinating Center at the University of South Florida College of Medicine. The Collagen Diagnostic Laboratory at the University of Washington served as the center for molecular and biochemical analyses. The respective institutional review boards of the participating clinical sites approved the study. Informed consent was obtained from all subjects or their legal guardians.

At the clinical sites, demographic data, medical history, and anthropometric measures of height, weight, and arm span were collected and recorded in a uniform fashion by trained personnel. Height, weight, and arm span were recorded as single measurements. The data were collected at every clinical site in accordance with the instructions outlined in the Manual of Operations developed for the multi-site study, thus ensuring consistency between the clinical sites. Parameters were reported using online case report forms as follows.

- Height was defined as the vertical distance between the crown of the head and soles of the feet. It was measured using a wall-mounted stadiometer and recorded to the nearest $0.1 \mathrm{~cm}$. When participants could not stand, the supine length was measured from the heels to the top of the head.

- Weight was measured on an upright calibrated digital or beam scale to the nearest $0.1 \mathrm{~kg}$. In individuals who were too young to stand alone, an infant scale was used. When children could not stand on the scales, they were weighed while being held by a parent, and the weight of the child was calculated by subtracting the weight of the parent from the total weight. The body mass index (BMI) was calculated as weight $(\mathrm{kg}) /$ height $(\mathrm{m})^{2}{ }^{2}$

- Arm span was measured as the distance from one furthermost fingertip to the other furthermost fingertip when the participant's arms were stretched out horizontally, using a nonstretching long measuring tape to the nearest $0.1 \mathrm{~cm}$. Arm span was measured as a single measure, as opposed to parts then added together.

Overall, 552 participants were enrolled, including 244 with OI type I, 110 with OI type III, 150 with OI type IV, 15 with OI type V, 12 with OI type VI, 5 with OI type VII, and 16 with an unclassified type of OI . The classification of OI was based on clinical features outlined in the Manual of Operations; however, genotypic information was used to reclassify patients when available. Data collected for analyses included age at enrollment, sex, OI type, family history of OI, history of bisphosphonate use (yes or no), history of rodding (yes or no), self-reported parental height, subject height, weight, and arm span. To analyze height within particular genotypes, we classified the type I collagen pathogenic variant (PV) as glycine substitution PV within the triple helix domain $(n=160)$, loss-of-function (nonsense, deletion, frameshift, or splicing; $n=144) \mathrm{PV}$ and nonglycine missense variations $(n=9)$. Given the small number of individuals with OI types V, VI, and VII, they were excluded from further formal statistical evaluations. The height, weight, and BMI data presented here were collected at the initial enrollment visit.

\section{Statistical analysis}

For participants below 20 years of age (OI type I, $n=163$; OI type III, $n=83$; OI type IV, $n=123$ ), the Centers for Disease Control and Prevention's (CDC's) growth curves were utilized to plot the height, weight, and BMI. The $\mathrm{z}$-scores were calculated using the L, M, and S parameters based on the 
Table 1 Characteristics of individuals with Ol enrolled in the Linked Clinical Research Centers

\begin{tabular}{|c|c|c|c|c|c|c|c|c|}
\hline & Ol I & OI III & OI IV & OI V & OI VI & OI VII & Unclassified & All \\
\hline Enrollment number & 244 & 110 & 150 & 15 & 12 & 5 & 16 & 552 \\
\hline Male, $n(\%)$ & $113(46.3)$ & $47(42.7)$ & $66(44)$ & $5(33.3)$ & $7(58.3)$ & $0(0)$ & $7(43.8)$ & 245 \\
\hline Female, $n(\%)$ & $131(53.7)$ & $63(57.3)$ & $84(56)$ & $10(66.7)$ & $5(41.7)$ & $5(100)$ & $9(56.3)$ & 307 \\
\hline Median age in years at enrollment (range) & $13.5(0-67)$ & $11.0(0-54)$ & $11.6(0-63)$ & $12.5(0-35)$ & $9.9(2-32)$ & $4.2(0-20)$ & $23.6(2-47)$ & 12.2 \\
\hline White, $n$ (\%) & $227(93)$ & $87(79.1)$ & $127(84.7)$ & $11(73.3)$ & $8(66.7)$ & $3(60)$ & $12(75)$ & 475 \\
\hline Black, $n(\%)$ & $7(2.9)$ & $10(9.1)$ & $14(9.3)$ & $2(13.3)$ & $0(0)$ & $0(0)$ & $2(12.5)$ & 35 \\
\hline Other race, $n(\%)$ & $10(4.1)$ & $13(11.8)$ & $9(6)$ & $2(13.3)$ & $4(33.3)$ & $2(40)$ & $2(12.5)$ & 42 \\
\hline Family history, $n(\%)$ & $153(62.7)$ & $9(8.2)$ & $44(29.3)$ & $5(33.3)$ & $1(8.3)$ & $3(60)$ & $7(43.8)$ & 222 \\
\hline Molecular testing done, $n(\%)$ & $154(63)$ & $67(61)$ & $109(73)$ & $8(53)$ & $4(33)$ & $5(100)$ & & 347 \\
\hline
\end{tabular}

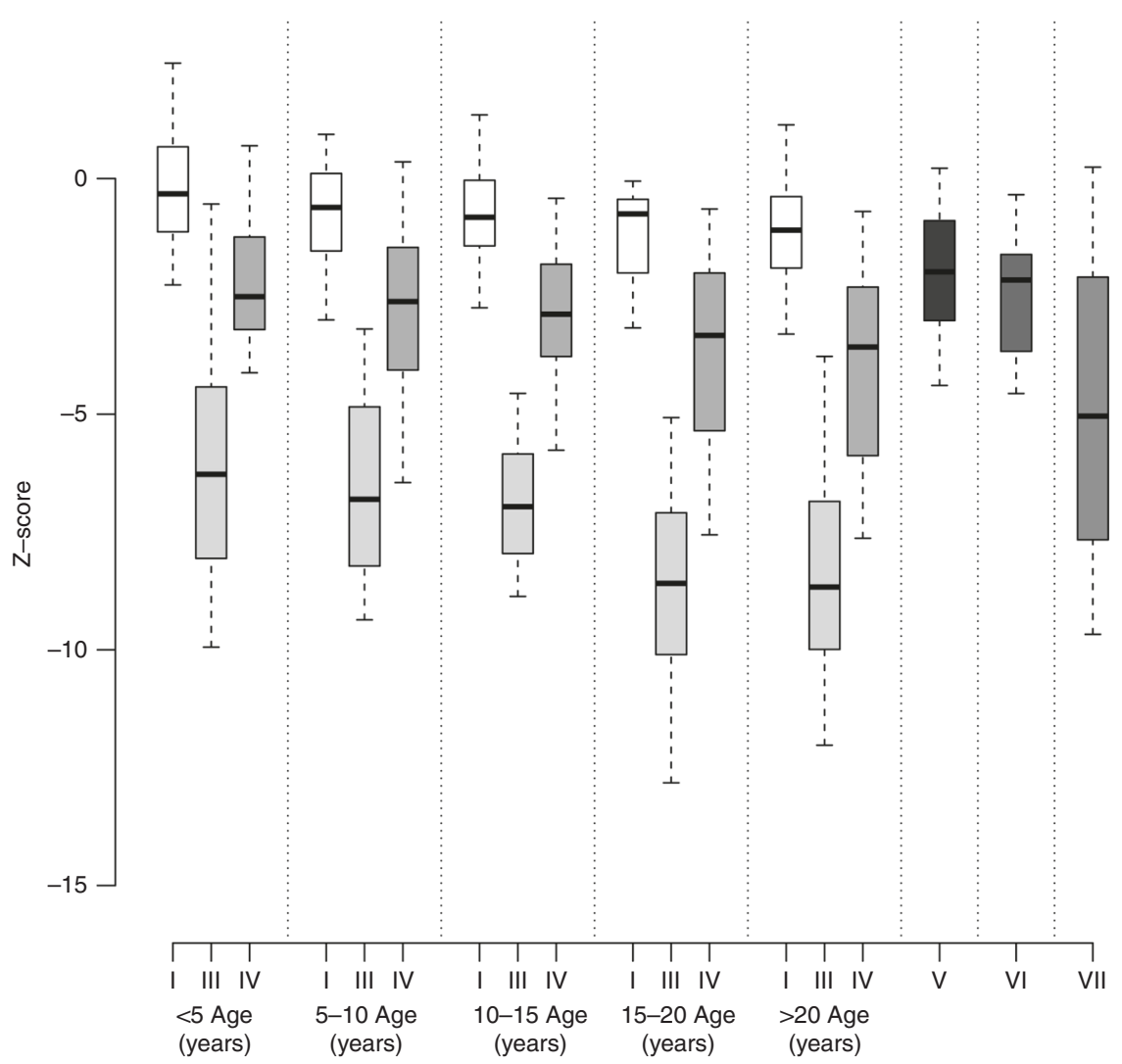

Fig. 1 Height z-scores by Ol subtype and age. Box plots of the median and interquartile range for height z-scores in the various age groups for type I, III, and IV OI. Z-scores are significantly lower for individuals with type III and type IV OI compared with type I OI. The number of participants in types V, VI, and VII were limited, and the pooled data are shown on the right

methodology described previously. ${ }^{15}$ The z-scores were computed using the AGD library in R. To compare z-scores for height, weight, and BMI between the OI types, a Komogrov-Smirnov test was performed to evaluate for normal distribution (analysis of variance, or analysis of variance on ranks, were used as appropriate). When comparing z-scores for height, weight, and BMI between OI types, we categorized individuals into age groups of $0-5$ years, $5-10$ years, $10-15$ years, $15-20$ years, and $>20$ years. Z-scores for height and weight for individuals $>20$ years of age were calculated using normative values from 20 -year-old individuals. The differences in proportions of individuals below the third percentile for height and weight between the OI types were analyzed using Fisher's exact tests. For adult participants in the study (OI type I, $n=81$; OI type III, $n=27$; and OI type IV, $n=27$ ) the expected midparental height was calculated from self-reported parental heights, and $5 \mathrm{~cm}$ was considered to be $1 \mathrm{SD}$ from the mean, as previously published. ${ }^{16}$ The differences in proportions of individuals with final heights below 2 SD from the expected height were analyzed using Fisher's exact tests. The growth velocity between OI subtypes was assessed, and the proportion of individuals falling outside of 2 SD from the mean based on normative data was ascertained. ${ }^{17}$ 

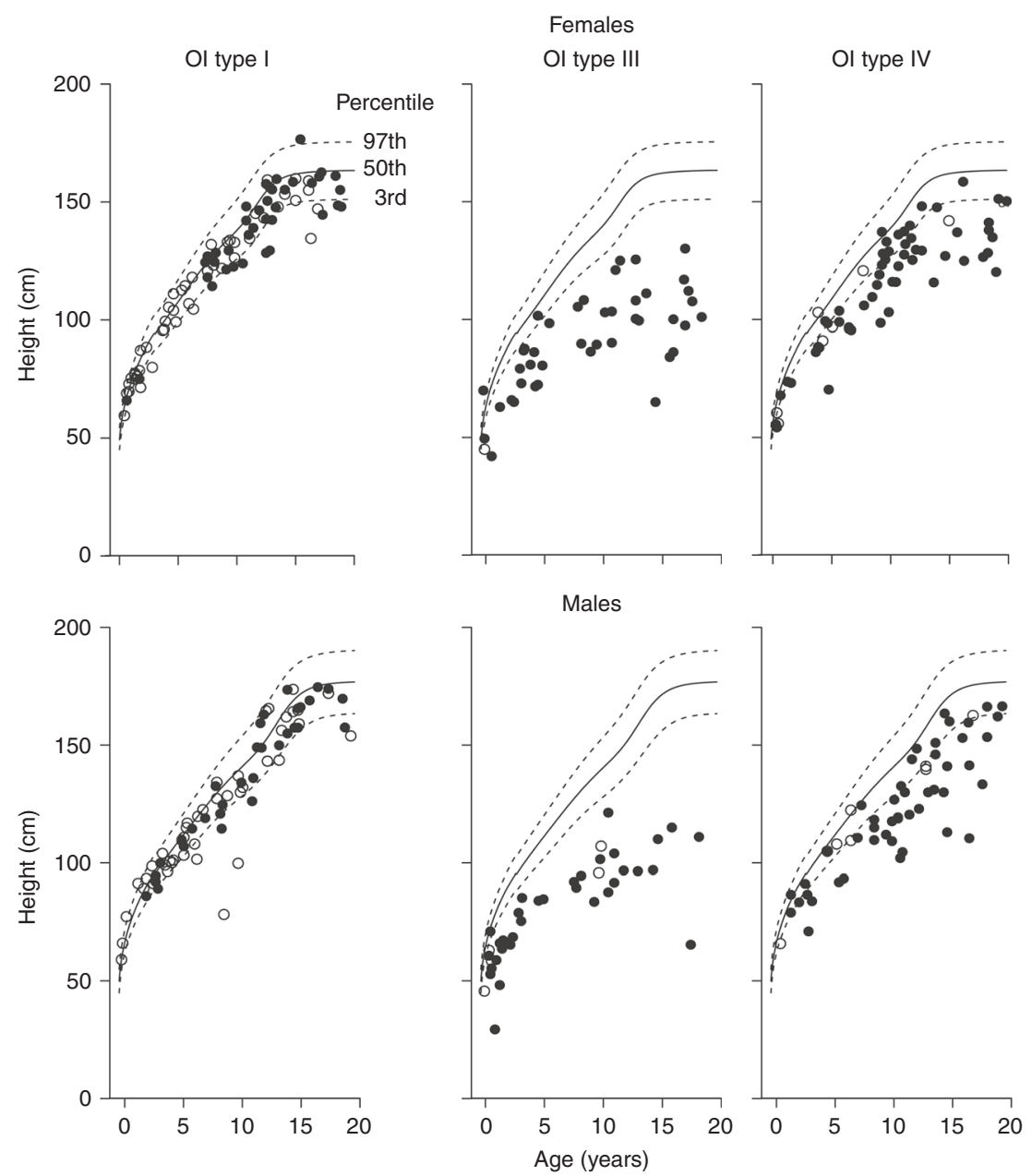

Fig. 2 Cross-sectional height measurements plotted on CDC growth curves. Each circle represents one participant. Open circles represent individuals who were naïve to bisphosphonate treatment, while black circles represent individuals who had received bisphosphonate at some point in their life

To further evaluate the potential effects of age, history of orthopedic rodding, history of bisphosphonate use, and sex on height, we performed generalized linear model analyses using a backward elimination strategy with the 'step' function in R. The height $\mathrm{z}$-score was the dependent variable, while the independent categorical variables included OI type (type I, III, or IV), sex (male or female), history of rodding (yes or no), and history of bisphosphonate therapy (yes or no); age was included as a continuous numeric variable. $P$ values were calculated using the likelihood ratio test, and correlations present with $P<0.05$ were tested for evidence of interaction.

The arm-span-to-height ratios between OI subtypes were compared by one-way analysis of variance among the age groups $<10$ years, $10-20$ years, and $>20$ years. These age categories were chosen based on previous normative data that show an increase in the arm-span-to-height ratio after 10 years of age. ${ }^{18}$

\section{RESULTS}

The characteristics of individuals enrolled in the study are outlined in Table 1. The autosomal dominant forms of type I collagen-related OI accounted for over $90 \%$ of all enrolled individuals.

\section{Height in OI}

In the pediatric population (age <20 years), the median (interquartile range (IQR)) z-scores for height in OI types I, III, IV, V, and VI were $-0.66(-1.43$ to -0.02$),-6.91(-8.41$ to -5.17$),-2.79$ ( -3.95 to -1.69$),-1.65(-2.16$ to -0.77$)$, and $-1.72(-2.36$ to -1.38$)$, respectively (Figure 1$)$. As expected, for the type I collagen-related OI across all age groups, individuals with OI type III had diminished height compared with OI type I and OI type IV, and individuals with OI type IV had decreased height compared with OI type I $(P<$ 0.05). When plotted on CDC growth curves, $9.5 \%$ of males and $18.1 \%$ of females with OI type I, $97.4 \%$ of males and $95.5 \%$ of females with OI type III, and $70.4 \%$ of males and $68.9 \%$ of females with OI type IV were below the third percentile for height (Figure 2). The odds ratio (95\% confidence interval) for being below the third percentile was 219 (50.5-953.4) for OI type III $(P<0.001$ compared with type I) and $11.7(6.5-20.9)$ for OI type IV $(P<0.001$ compared with type I). For OI type III, the odds ratio for being below the third percentile was 18.76 (4.37-80.45; $P<0.001$ compared with OI type IV).

In adults with OI, the median (IQR) z-scores for height were -1.10 ( -1.90 to -0.38$),-8.67$ ( -9.99 to -6.85$)$, and 
$-3.58(-5.88$ to -2.30$)$ for OI types I, III, and IV, respectively. Pairwise comparisons revealed significant differences between OI subtypes $(P<0.05)$. In adults without a family history of OI, we calculated the expected midparental height based on the self-reported parental height. The final adult height was affected in all OI subtypes, with $48 \%$ with OI type I, $100 \%$ with OI type III, and 58\% with OI type IV below 2 SD from the expected midparental height. A Fisher's exact test demonstrated a severe reduction in the final adult height for type III compared with type IV $(P<0.001)$ and type I OI $(P<0.01)$, but no statistically significant difference was noted between OI types I and IV.

We correlated the height $\mathrm{z}$-scores with PV type in individuals who had demonstrated pathogenic variants in either $C O L 1 A 1$ or $C O L 1 A 2$. For individuals with glycine substitution PV the median height $\mathrm{z}$-score (IQR) was -4.7 $(-6.7$ to -2.1$)$; for individuals with other missense variants, it was $-3.5(-1.7$ to -5.2$)$; and for loss-of-function variants, it was $-1.28(-2.2$ to -0.72$)$. Height was significantly diminished in individuals with glycine substitutions compared with loss of function $(P<0.001)$. Furthermore, we examined whether different glycine substitutions had an effect on height. Individuals with glycine-to-serine substitutions had a median height $\mathrm{z}$-score of $-5.6(-6.7$ to -3.1$)$, whereas the median heights in glycine-to-aspartate and glycine-toanother-amino-acid substitutions were $-3.5(-5.5$ to -3.2$)$ and $-3.2(-6.8$ to -1.7$)$, respectively. While the median was lower for glycine-to-serine substitutions, this difference did not reach statistical significance.

\section{Growth velocity in OI}

We used the longitudinal aspect of the cohort to examine growth velocity in participants between the ages of 3 and 16 years. We selected individuals who had at least two height measurements separated by 6 to 18 months. The velocity of growth was calculated in $\mathrm{cm} \bullet y_{e a r^{-1}}$ and the value obtained was plotted against normative data. In individuals with three or more measurements, the values between two consecutive measurements were considered as distinct data points and thus plotted separately. In total, 127 individuals with OI type I ( $n=61$ male; $n=66$ female; $n=259$ growth velocity measurements), 46 individuals with OI type III ( $n=20$ male; $n=26$ female, $n=79$ growth velocity measurements), and 76 individuals with OI type IV $(n=37$ male, $n=39$ female,
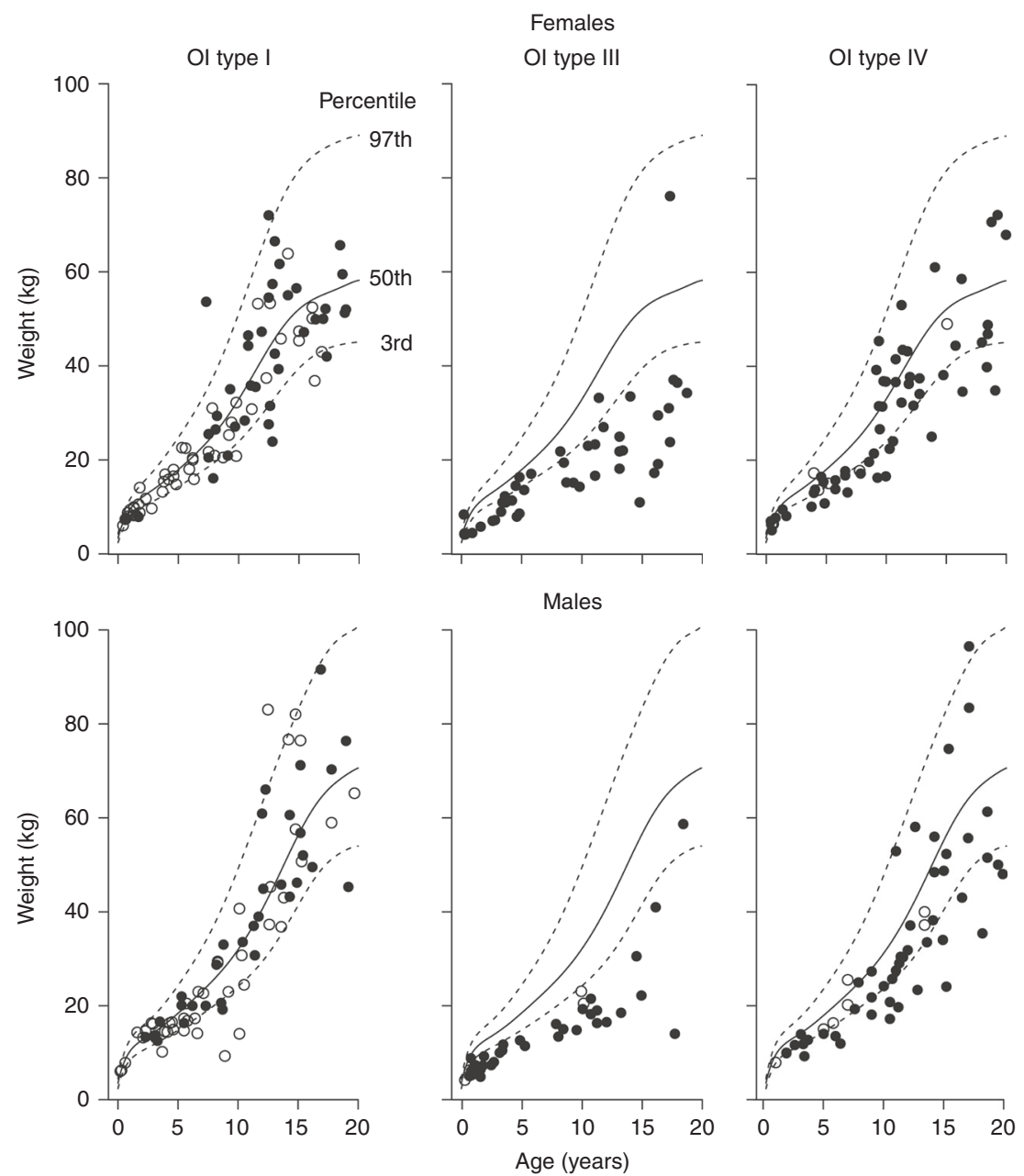

Fig. 3 Cross-sectional weight measurements plotted on CDC growth curves. Each circle represents one participant. Open circles represent individuals who were naïve to bisphosphonate treatment, while black circles represent individuals who had received bisphosphonate at some point in their life 


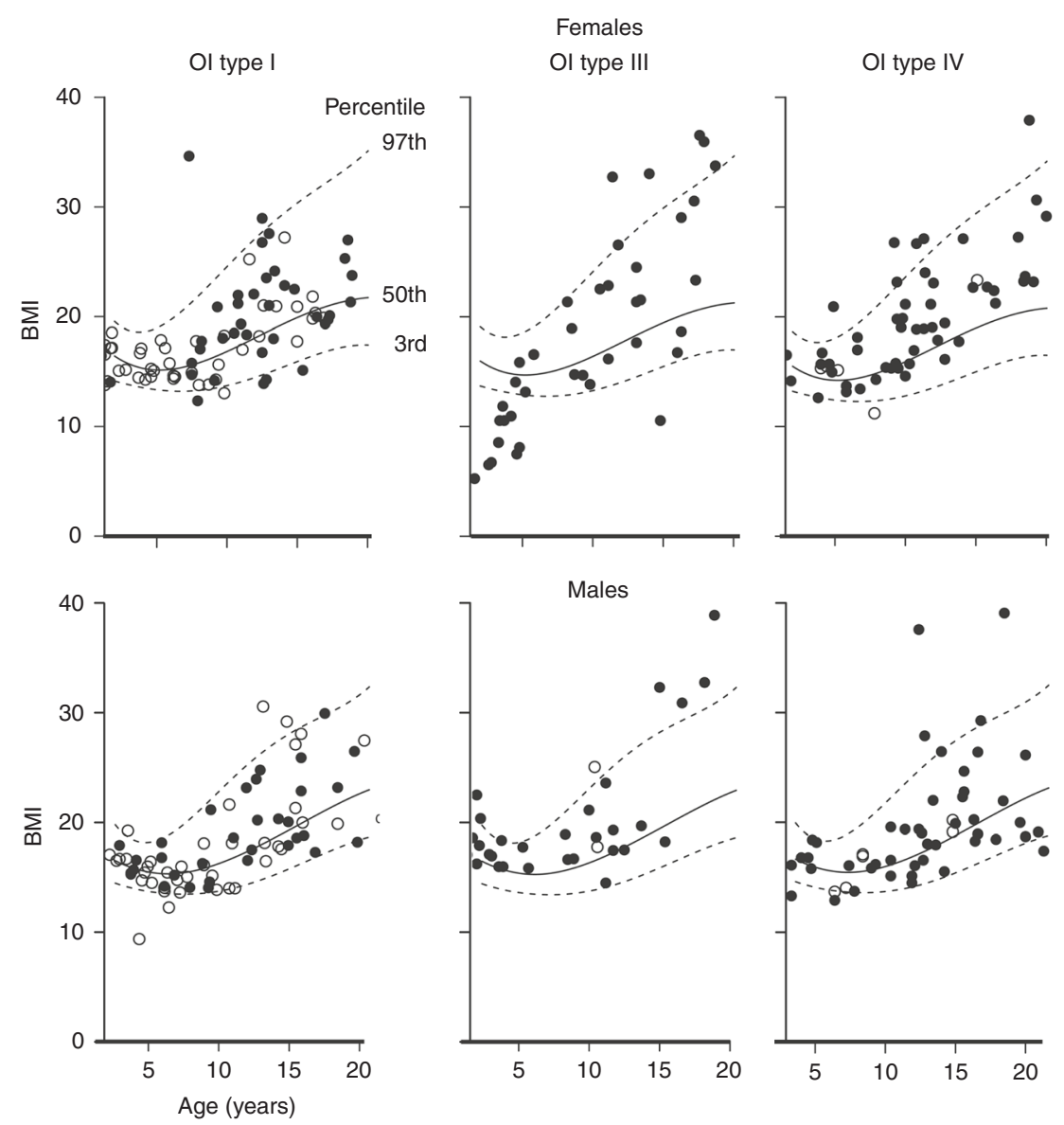

Fig. 4 Cross-sectional BMI measurements plotted on CDC growth curves. Open circles represent individuals who were naïve to bisphosphonate treatment, while black circles represent individuals who had received bisphosphonate at some point in their life

$n=148$ growth velocity measurements) were included in these analyses. In OI type I, $12.4 \%$ of growth velocities (32/ $259)$ were 2 SD below the mean or lower for growth velocity, whereas in OI types III and IV, the proportions were $51.9 \%$ $(41 / 79)$ and $31.1 \%$ (46/148), respectively. This corresponds to an increased odds ratio (95\% confidence interval) of having diminished growth velocity of $7.65(4.30-13.61)$ in OI type III compared with type I, and of $3.20(1.92-5.32)$ in OI type IV compared with type I $(P<0.0001)$.

\section{Weight in OI}

The median (IQR) z-scores for weight in the pediatric population for OI types I, III, IV, V, and VI were -0.41 $(-1.14$ to 0.56$),-4.55$ ( -6.40 to -2.85$),-1.55(-2.37$ to $-0.31),-0.31(-1.81$ to 0.32$)$, and $-0.99(-1.68$ to 0.08$)$, respectively (Supplementary Figure S1 online). Analysis of variance on ranks demonstrated significant differences between the OI subtypes across all age groups, with weight in OI type III being significantly lower than in OI types I and IV $(P<0.05)$. We observed significant differences for weight between OI types IV and I only in the age groups $0-5$ years and $10-15$ years $(P<0.05)$. As for height, a similar relationship was seen for weight, where $11.8 \%$ of males and $16.9 \%$ of females with OI type I, $35.8 \%$ of males and $33.3 \%$ of females with OI type IV, and $94.7 \%$ of males and $84.8 \%$ of females with OI type III were below the third percentile $(P<0.001$; Figure 3). The odds ratios ( $95 \%$ confidence interval) for being below the third percentile were $43.20(19.50-95.69)$ for OI type III $(P<0.001$ compared with type I) and $15.76(7.32-$ 33.95) for OI type IV $(P<0.001$ compared with type I). For individuals with OI type III compared with OI type IV, the odds ratio for being below the third percentile was 2.74 (1.524.94; $P<0.005)$.

\section{BMI in OI}

The median (IQT) z-scores for BMI in the pediatric population for OI types I, III, and IV were 0.10 ( -0.58 to 0.94$), 0.91(0.42$ to 1.61 ) and 0.67 ( -0.18 to 1.33 ) (Figure 4 and Supplementary Figure S2 online). When BMI was calculated using measured height, individuals with OI type III had increased BMI compared with OI types I and IV in adults and children in the age groups $5-10$ years and $15-20$ years $(P<0.05)$.

\section{Arm-span-to-height ratio in $\mathrm{OI}$}

The arm-span-to-height ratio is an indicator of the relative severity of linear growth abnormalities of the axial and/or 
lower limb compared with the upper limbs. The adult arm span is typically $5 \mathrm{~cm}$ greater in males and $2 \mathrm{~cm}$ greater in females compared with height. ${ }^{18}$ Arm-span-to-height ratios change with age; i.e., the arm span is less than height in early childhood, equal to height by about 10 years in males and 12 years in females, and greater than height thereafter. ${ }^{18}$ Thus, we compared the arm-span-to-height ratio in the age groups $<10$ years, $10-20$ years, and $>20$ years. Only in the $>20$ years group did we observe significant increased arm-span-toheight ratios in OI types III and IV compared with type I $(P<$ 0.001 and $P<0.05$, respectively) (Supplementary Figure S3 online).

\section{Potential correlates affecting height in OI}

Previous studies have shown that individuals with severe forms of OI can have "flattening" of their height curves so that the height $\mathrm{z}$-scores worsen with age. ${ }^{7,8} \mathrm{With}$ the widespread use of bisphosphonates, the effect of the medications on linear growth has been assessed in short-term studies without conclusive answers. ${ }^{19-23}$ To understand the correlations between some covariates and the height z-scores, we performed a generalized linear model analysis. As expected, OI subtype was strongly correlated with the height $\mathrm{z}$-scores through all pediatric ages $(P<0.001)$. Increasing age correlated with reduced height $\mathrm{z}$-scores $(P<0.05)$. Independent correlations were also observed for history of rodding $(P<$ $0.01)$ and use of bisphosphonates $(P<0.05)$, where both of these covariates correlated with overall reduced $z$-scores. However, there were significant interactions between the OI subtype, history of rodding, and bisphosphonate use. Thus, we could not independently assess the effects of these covariates on height.

\section{DISCUSSION}

OI is a clinically heterogeneous disorder characterized by an increased predisposition to recurrent fractures and bone deformities. ${ }^{3}$ Although it is well recognized that growth deficiency can be found in patients with severe forms of OI, there is a lack of large-scale studies on growth parameters in various subtypes of OI. In this study, we included a large cohort of children and adults with OI from multiple clinical centers in North America.

The height measurements in this cohort are consistent with the expected phenotypes for OI and growth patterns observed in other studies. ${ }^{3,5-8}$ Height in OI type III is more severely affected compared with OI types I and IV. Although some individuals with OI types I and IV can have heights within the normal range, the overall adult height is affected in all subtypes. Even in OI type I (the mildest form), the final height in nearly half of individuals is $2 \mathrm{SD}$ below the expected midparental height. Given these distinct patterns of height, height $\mathrm{z}$-scores could be utilized for clinical classification of type I collagen-related OI.

The etiology for short stature in OI is multifactorial. Scoliosis, kyphosis, vertebral fractures, recurrent long bone fractures, and bone deformities are important contributing factors. The incidence of scoliosis and the rate of progression of curvature are significantly higher in the more severe forms of OI. ${ }^{24}$ In a retrospective study of over 300 children with OI, Anissipour and colleagues found that the rates of progression of scoliosis were $6^{\circ}, 4^{\circ}$, and $1^{\circ}$ in OI types III, IV, and I, respectively. Spinal deformities in the severe forms of OI can lead to a reduction in truncal height. Similarly, fractures and bowing of the femur and tibia, fractures occurring through the growth plates, and abnormalities of the epiphyses in the severe forms of OI lead to decreased limb length and overall height. The varying severity of involvement of the axial and appendicular skeleton can be reflected by the arm-span-toheight ratio. In this study, we observed that the arm-span-toheight ratio was increased in adults with types III and IV OI compared with type I. These results are consistent with previous observations. ${ }^{6,8}$ Collectively, these findings suggest that truncal height is relatively more reduced than the length of the long bones in the more severe forms of OI.

However, clinical experience and previous studies have demonstrated that overall height can be affected even in individuals without significant bone deformities. ${ }^{7,8}$ Thus, it has been hypothesized that the primary matrix and cellular abnormalities may have a role in the decreased growth rates in OI. Decreased responsiveness to growth hormone and insulin-like growth factor 1 has been suggested as one of the mechanisms in a few studies. ${ }^{25,26}$ More recently, it was discovered that excessive transforming growth factor-beta (TGF- $\beta$ ) signaling is an important driver of the bone and extraskeletal abnormalities in moderate-to-severe forms of OI. ${ }^{27}$ The collagen overmodification in severe forms of OI affects the interaction of type I collagen with small leucinerich proteoglycans that bind TGF- $\beta$, which results in increased availability of the ligand. ${ }^{27-30}$ Increased TGF- $\beta$ signaling in the growth plate could affect bone growth. In a mouse model of E-selectin ligand (a negative regulator of TGF- $\beta$ ) deficiency, Yang and colleagues demonstrated reduced chondrocyte proliferation and delayed terminal differentiation. ${ }^{31}$ Thus, it is possible that increased TGF- $\beta$ signaling could contribute to the abnormal growth in OI. While final adult height is affected across all types of OI, using the longitudinal nature of the dataset, we also demonstrate that the growth velocity is affected. Current information on growth velocity in OI patients is rather limited, and studies that have addressed this have done so in the context of therapy. ${ }^{11,32,33}$ It is not known whether the decreased growth velocity in OI type III and IV is driven by the recurrent fractures or intrinsic abnormalities of the bone and cartilage.

Bisphosphonates are currently considered a standard-ofcare and are widely used for the treatment of individuals with OI, but the effects of bisphosphonates on fracture risk in OI are difficult to address and the results have been inconclusive. $^{19-23,33-37}$ Few studies have addressed the effects of bisphosphonates on height in OI. Zeitlin and colleagues showed that four years of therapy with pamidronate increased height $\mathrm{z}$-scores in children with moderate-to-severe OI. ${ }^{11}$ Similarly, it has also been shown that bisphosphonate therapy 
decreases the rate of progression of scoliosis in type III OI if treatment is started before the age of six years; ${ }^{24}$ however, recent meta-analyses have not shown a significant effect of bisphosphonates on height. ${ }^{38,39}$ In this study, we tried to find correlations between height $\mathrm{z}$-scores and the use of bisphosphonates. In our cohort, $30 \%$ of individuals with OI type I, $84 \%$ with OI type III, and $76 \%$ with OI type IV had received intravenous bisphosphonate at some time, and $15 \%$ in each subtype had received oral bisphosphonates. However, on generalized linear model analyses, there was a significant interaction between the OI subtype and the use of bisphosphonates, and the proportion of individuals with severe OI who were naïve to bisphosphonates was very low, precluding independent assessment of the effect of bisphosphonates on height.

Weight in OI has not been studied as extensively as height. In this report, we observed that individuals with OI type III continue to fall below the third percentile of the CDC growth curves across all age groups. These results are consistent with previous publications reporting that individuals with severe OI are underweight. ${ }^{6}$ However, in spite of the lower weight, the calculated BMI in OI tends to be higher, especially in OI type III. Using peripheral quantitative computed tomography, Palomo and colleagues showed that the fat cross-sectional area at the forearm in individuals with $\mathrm{OI}$ is similar to the control population. ${ }^{40}$ These data imply that "increased BMI" is a result of the small value of the denominator during calculation and may not portend increased metabolic risks. It may thus be more important and practical to monitor serial weight in OI, especially in adults. Future studies that systematically measure body composition in OI may be helpful in risk stratification and further management.

Disorder-specific growth charts can be very helpful in monitoring growth in the clinic. To our knowledge, the only growth charts that have been developed in OI are for OI type I. ${ }^{13}$ One challenge in creating standard growth charts for all subtypes of OI is the great variability in the clinical presentations. The experience gained for the studies in the OI LCRC was pivotal in establishing the Brittle Bone Disorders Consortium (BBDC). The data being collected in the BBDC would be helpful in generating OI-specific growth curves for all subtypes of OI.

In summary, our analyses of the largest cohort of individuals with OI demonstrates that individuals with severe forms of OI have reduced height as well as weight compared with those with type I OI. This important study is a significant first step in the construction of growth curves for this disorder.

\section{SUPPLEMENTARY MATERIAL}

Supplementary material is linked to the online version of this article at https://doi.org/10.1038/s41436-018-0045-1.

\section{ACKNOWLEDGMENTS}

This work was supported by the BBDC (1U54AR068069-0), a part of the National Center for Advancing Translational Sciences' Rare
Diseases Clinical Research Network. BBDC is funded through a collaboration between the Office of Rare Diseases Research of the National Center for Advancing Translational Sciences, National Institute of Arthritis and Musculoskeletal and Skin Diseases, and National Institute of Dental and Craniofacial Research. The content of this paper is solely the responsibility of the authors and does not necessarily represent the official views of the National Institutes of Health. The BBDC is also supported by the Osteogenesis Imperfecta Foundation. This work was supported by the Clinical Translational Core of the Baylor College of Medicine Intellectual and Developmental Disabilities Research Center (1U54HD083092) from the Eunice Kennedy Shriver National Institute of Child Health and Human Development. S.B. was supported by the Texas Department of Health Services. M.J. and A.T. were supported by T32GM07526-40. This work was supported by the Doris Duke Charitable Foundation (grant 2013095 to S.C.S.N.). We acknowledge the clinical research teams: M. Mullins, A. Tran, and S. Carter (Baylor College of Medicine), V. Vensel, J. Christie, and A. Hata (Oregon Health and Science University), M. Durigova (Shriners Hospital for Children), and L. Davey (Alfred I. duPont Hospital for Children). The authors acknowledge the contributions of the members of the BBDC: David R. Eyre, PhD, Department of Orthopedic and Sports Medicine, University of Washington, Seattle, Washington, USA; Deborah Krakow, MD, Department of Orthopedic Surgery, David Geffen School of Medicine, University of California, Los Angeles, Los Angeles, California, USA; Laura Tosi, MD, Bone Health Program, Children's National Health System, Washington, DC, USA; Cathleen L. Raggio, MD, Hospital for Special Surgery, New York, USA; Eric S. Orwoll, MD, Department of Medicine, Division of Endocrinology, Oregon Health Sciences University, Portland, Oregon, USA; and Eric T. Rush, MD, University of Nebraska Medical Center, Omaha, Nebraska, USA.

\section{MEMBERS OF THE BRITTLE BONE DISEASE CONSORTIUM}

David R Eyre, Deborah Krakow, Laura Tosi, Cathleen L Raggio, Eric S Orwoll, and Eric T Rush.

${ }^{12}$ Department of Orthopedic and Sports Medicine, University of Washington, Seattle, Washington, USA; ${ }^{13}$ Department of Orthopedic Surgery, David Geffen School of Medicine, University of California, Los Angeles, Los Angeles, California, USA; ${ }^{14}$ Bone Health Program, Children's National Health System, Washington DC, USA; ${ }^{15}$ Hospital for Special Surgery, New York, USA; ${ }^{16}$ Department of Medicine, Division of Endocrinology, Oregon Health Sciences University, Portland, Oregon, USA; ${ }^{17}$ University of Nebraska Medical Center, Omaha, Nebraska, USA

\section{DISCLOSURE}

The authors declare no conflicts of interest.

\section{REFERENCES}

1. Rauch F, Glorieux FH. Osteogenesis imperfecta. Lancet. 2004;363:137785. 
2. Sykes $B$, Wordsworth $P$, Ogilvie $D$, et al. Osteogenesis imperfecta is linked to both type I collagen structural genes. Lancet. 1986;328:69-72.

3. Sillence DO, Senn A, Danks DM. Genetic heterogeneity in osteogenesis imperfecta. J Med Genet. 1979;16:101-16.

4. Bellur $\mathrm{S}$, Jain $\mathrm{M}$, Cuthbertson $\mathrm{D}$, et al. Cesarean delivery is not associated with decreased at-birth fracture rates in osteogenesis imperfecta. Genet Med. 2016;18:570-6.

5. Vetter U, Pontz B, Zauner E, et al. Osteogenesis imperfecta: a clinical study of the first ten years of life. Calcif Tissue Int. 1992;50:36-41.

6. Aglan MS, Zaki ME, Hosny L, et al. Anthropometric measurements in Egyptian patients with osteogenesis imperfecta. Am J Med Genet A. 2012:158 A:2714-8.

7. Germain-Lee EL, Brennen FS, Stern D, et al. Cross-sectional and longitudinal growth patterns in osteogenesis imperfecta: implications for clinical care. Pediatr Res. 2015;79:489-95.

8. Lund A, Müller J, Skovby F. Anthropometry of patients with osteogenesis imperfecta. Arch Dis Child. 1999;80:524-8.

9. Engelbert RH, Gerver WJ, Breslau-Siderius $\amalg$, et al. Spinal complications in osteogenesis imperfecta: 47 patients 1-16 years of age. Acta Orthop Scand. 1998;69:283-6.

10. Engelbert RH, Uiterwaal CS, Gerver WJ, et al. Osteogenesis imperfecta in childhood: impairment and disability. A prospective study with 4-year follow-up. Arch Phys Med Rehabil. 2004;85:772-8.

11. Zeitlin L, Rauch F, Plotkin $H$, et al. Height and weight development during four years of therapy with cyclical intravenous pamidronate in children and adolescents with osteogenesis imperfecta types I, III, and IV. Pediatrics. 2003;111:1030-6.

12. Hamza RT, Abdelaziz TH, Elakkad M. Anthropometric and nutritional parameters in Egyptian children and adolescents with osteogenesis imperfecta. Horm Res Paediatr. 2015;83:311-20.

13. Graff K, Syczewska M. Developmental charts for children with osteogenesis imperfecta, type I (body height, body weight and BMI). Eur J Pediatr. 2017:176:311-6.

14. Patel RM, Nagamani SCS, Cuthbertson D, et al. A cross-sectional multicenter study of osteogenesis imperfecta in North America-results from the Linked Clinical Research Centers. Clin Genet. 2015:87:133-40.

15. Kuczmarski RJ, Ogden CL, Guo SS, et al. 2000 CDC growth charts for the United States: methods and development. Vital Health Stat. 2002; 11:1-190.

16. Nwosu BU, Lee MM. Evaluation of short and tall stature in children. Am Fam Physician. 2008;78:597-604.

17. Tanner JM, Davies PS. Clinical longitudinal standards for height and height velocity for North American children. J Pediatr. 1985;107:317-29.

18. Belt-Niedbala BJ, Ekvall S, Cook CM, et al. Linear growth measurement: a comparison of single arm-lengths and arm-span. Dev Med Child Neurol. 1986;28:319-24.

19. Bishop N, Adami S, Ahmed SF, et al. Risedronate in children with osteogenesis imperfecta: a randomised, double-blind, placebo-controlled trial. Lancet. 2013;382:1424-32.

20. Rauch F, Munns CF, Land C, et al. Risedronate in the treatment of mild pediatric osteogenesis imperfecta: a randomized placebo-controlled study. J Bone Miner Res. 2009:24:1282-9.

21. Sakkers $R$, Kok $D$, Engelbert $R$, et al. Skeletal effects and functional outcome with olpadronate in children with osteogenesis imperfecta: a 2-year randomised placebo-controlled study. Lancet. 2004;363:1427-31.

22. Seikaly MG, Kopanati S, Salhab N, et al. Impact of alendronate on quality of life in children with osteogenesis imperfecta. I Pediatr Orthop. 2005;25:786-91.
23. Letocha $A D$, Cintas $H L$, Troendle JF, et al. Controlled trial of pamidronate in children with types III and IV osteogenesis imperfecta confirms vertebral gains but not short-term functional improvement. J Bone Miner Res. 2005;20:977-86.

24. Anissipour AK, Hammerberg KW, Caudill A, et al. Behavior of scoliosis during growth in children with osteogenesis imperfecta. J Bone Joint Surg Am. 2014;96:237-43.

25. Marini JC, Bordenick S, Heavner G, et al. Evaluation of growth hormone axis and responsiveness to growth stimulation of short children with osteogenesis imperfecta. Am J Med Genet. 1993:45:261-4.

26. Marini JC, Bordenick S, Heavner G, et al. The growth hormone and somatomedin axis in short children with osteogenesis imperfecta. J Clin Endocrinol Metab. 1993;76:251-6.

27. Grafe I, Yang T, Alexander S, et al. Excessive transforming growth factor$\beta$ signaling is a common mechanism in osteogenesis imperfecta. Nat Med. 2014;20:670-5.

28. Keene DR, San Antonio JD, Mayne R, et al. Decorin binds near the C terminus of type I collagen. J Biol Chem. 2000;275:21801-4.

29. Takeuchi $Y$, Kodama $Y$, Matsumoto $T$. Bone matrix decorin binds transforming growth factor-beta and enhances its bioactivity. I Biol Chem. 1994:269:32634-8.

30. Markmann A, Hausser $H$, Schönherr $E$, et al. Influence of decorin expression on transforming growth factor-beta-mediated collagen gel retraction and biglycan induction. Matrix Biol. 2000;19:631-6.

31. Yang $T$, Grafe I, Bae $Y$, et al. E-selectin ligand 1 regulates bone remodeling by limiting bioactive TGF- $\beta$ in the bone microenvironment. Proc Natl Acad Sci. 2013;110:7336-41.

32. Land C, Rauch F, Munns CF, et al. Vertebral morphometry in children and adolescents with osteogenesis imperfecta: effect of intravenous pamidronate treatment. Bone. 2006;39:901-6.

33. Glorieux FH, Bishop NJ, Plotkin $H$, et al. Cyclic administration of pamidronate in children with severe osteogenesis imperfecta. $N$ Engl $J$ Med. 1998;339:947-52.

34. Dimeglio LA, Ford L, McClintock C, et al. A comparison of oral and intravenous bisphosphonate therapy for children with osteogenesis imperfecta. J Pediatr Endocrinol Metab. 2005;18:43-53.

35. Gatti $D$, Antoniazzi $F$, Prizzi $R$, et al. Intravenous neridronate in children with osteogenesis imperfecta: a randomized controlled study. J Bone Miner Res. 2004:20:758-63.

36. Barros ER, Saraiva GL, de Oliveira TP, et al. Safety and efficacy of a 1-year treatment with zoledronic acid compared with pamidronate in children with osteogenesis imperfecta. J Pediatr Endocrinol Metab. 2012;25:48591.

37. Bishop N, Harrison R, Ahmed F, et al. A randomized, controlled doseranging study of risedronate in children with moderate and severe osteogenesis imperfecta. J Bone Miner Res. 2010;25:32-40.

38. Hald JD, Evangelou E, Langdahl BL, et al. Bisphosphonates for the prevention of fractures in osteogenesis imperfecta: meta-analysis of placebo-controlled trials. J Bone Miner Res. 2015;30:929-33.

39. Dwan K, Phillipi CA, Steiner RD, et al. Bisphosphonate therapy for osteogenesis imperfecta. Cochrane Database Syst Rev. 2014;23: CD005088.

40. Palomo T, Glorieux FH, Schoenau E, et al. Body composition in children and adolescents with osteogenesis imperfecta. J Pediatr. 2016; 169:232-7. 